ÉGYPTE monde arabe

\section{Égypte/Monde arabe}

23 | 1995

Géographies de l'Égypte 2

\title{
Régularisation foncière, propriété et espace urbanisé
}

\section{Agnès Deboulet}

\section{(2) OpenEdition}

\section{Journals}

Édition électronique

URL : https://journals.openedition.org/ema/965

DOI : $10.4000 /$ ema.965

ISSN : 2090-7273

\section{Éditeur}

CEDEJ - Centre d'études et de documentation économiques juridiques et sociales

\section{Édition imprimée}

Date de publication : 30 septembre 1995

Pagination : 57-76

ISSN : 1110-5097

\section{Référence électronique}

Agnès Deboulet, «Régularisation foncière, propriété et espace urbanisé », Égypte/Monde arabe [En ligne], 23 | 1995, mis en ligne le 08 juillet 2008, consulté le 07 juillet 2022. URL : http:// journals.openedition.org/ema/965; DOI : https://doi.org/10.4000/ema.965

Ce document a été généré automatiquement le 7 juillet 2022.

Tous droits réservés 


\title{
Régularisation foncière, propriété et espace urbanisé
}

\author{
Agnès Deboulet
}

1 Les études portant sur l'Égypte ou d'autres pays, africains ou latino-américains, mettent couramment l'accent sur les apports respectifs des visions officielles et des façons de faire populaires dans le façonnement de l'habitat. Eu égard à l'importance qu'ont prises aujourd'hui les extensions urbaines de création populaire, il y a un enjeu non moins important à comprendre de quelle façon émergent, se construisent, se consolident des représentations de l'espace mais aussi de la norme juridique, permettant aux classes populaires de s'installer en ville, d'y construire, de se forger une identité.

2 Comme la plupart des pays du tiers-monde, mais aussi certains pays de l'Europe du Sud, l'Égypte est confrontée à une situation critique en matière de logement. Les normes étatiques en matière de statut foncier, de permis de construire, de notion de territoire urbanisé, restent souvent extérieures aux pratiques. Sur le plan juridique, on parle dorénavant de «droit intermédiaire » ou de «droit populaire » pour désigner ces pratiques qui se trouvent à la lisière entre normes étatiques et pratiques coutumières. Sur le plan de l'habitat, certains auteurs parient de "métissage » des pratiques. Plutôt que de trouver une terminologie ad hoc, il me paraît plus pertinent de réfléchir à la façon dont se construisent ces représentations populaires et d'évaluer quelle distance les sépare des normes étatiques, en tenant compte du "pluralisme légal» qui caractérise à la fois le rapport de l'État et des citoyens au droit".

3 Je partirai donc du postulat que la dichotomie « légal/illégal », de même que celle qui oppose habitat majoritaire et habitat vernaculaire, correspond à deux réalités qui puisent bien souvent l'une dans l'autre. De plus, cette vision dichotomique tend à marginaliser des pratiques populaires devenues, en fait, largement majoritaires, et en minimise la complexité. Face à ce schéma d'interprétation dualiste, on peut constater que les normes populaires s'ajustent aux normes étatiques tout en préservant une marge de flexibilité. La question reste de savoir comment s'élaborent les définitions. Cela amène à s'interroger sur la capacité des acteurs, en cas d'intervention a posteriori 
des pouvoirs publics, à faire montre de leur compétence tout en instrumentalisant le sol en relation avec leurs habitus et aspirations. En filigrane, je m'efforce donc ici de reconstituer les logiques de l'action et des représentations présidant à l'appropriation et à l'urbanisation de terrains en Égypte, en m'appuyant sur des exemples tirés de l'occupation sans titre de terrains du domaine de l'État.

Les conflits fonciers : enjeux et perceptions réciproques des protagonistes

4 Dans un précédent article ${ }^{6}$ j'avais brièvement exposé comment la moitié sud de la zone archéologique de Fustat, située à proximité de la citadelle du Caire, avait été l'objet d'une invasion foncière conduisant à l'urbanisation totale du plateau. L'étude plus approfondie menée en 1991 montre en réalité que plusieurs types d'invasion foncière s'y superposent. Certaines prennent la forme de lotissements commerciaux, d'autres sont des invasions collectives ou individuelles opérées par des ménages en quête de logement.

Détaillons dans un premier temps la situation de confusion foncière qui a permis à des squatters groupés, à d'autres isolés et, dans les années récentes, à des lotisseurs, de s'installer sur un site à la fois très vaste (environ $200 \mathrm{ha}$ ) et à la localisation centrale. Ce sera l'occasion de voir que les pratiques illégales qui se développent dans une société en mal de logement reproduisent et distordent quelque peu des usages eux aussi répandus dans les sphères dirigeantes. Par ailleurs, nul ne parvient, dans ce cas, à démêler ce qui est du côté du droit et ce qui ne l'est pas. Au fond, on observe un parallélisme assez frappant entre l'usage du conflit foncier parmi les dirigeants et ce même usage au sein des classes populaires. Le conflit vise, de part et d'autre, la quête d'une rente foncière.

Une lutte pour le sol à armes inégales

6 Recouvrant des vestiges archéologiques fort importants (en particulier ceux de l'ancienne ville de Fustat), une partie non négligeable du plateau est sous la responsabilité de la direction des Antiquités, organisme dépendant du ministère de la Culture. Celui-ci dispose d'un droit d'usage qui ne vaut pas propriété. Jusqu'en 1972, le détenteur légal de ce terrain était le gouvernorat du Caire. A cette date, le gouvernement égyptien de l'époque décide d'aliéner ce patrimoine en cédant la plus grande portion de la partie sud du plateau, située en deçà de la route du Vieux-Caire, à une société publique dite "Société de Ma'âdi pour le logement et la promotion immobilière ». La direction des Antiquités semble alors farouchement opposée à ce transfert, arguant de l'impossibilité matérielle de fouiller l'ensemble du site avant sa mise en construction.

7 Il ressort clairement des entretiens que la surveillance des terrains organisée par ces trois institutions a été très insuffisante. Or, chacune des parties en présence rejette sur les autres la responsabilité du début de l'invasion en désignant, par exemple, la négligence des gardiens ou, à mots couverts, la corruption. Les uns justifient leur impéritie en évoquant la complicité active des autres dans l'installation des premiers squatters. Ajoutons au tableau la présence, assez ostentatoire par endroits, de militaires lotisseurs et surtout, l'implantation non négociée d'un camp de la police montée, à proximité de Basatîn?

8 Deux ingrédients élémentaires de la situation de blocage foncier permettant l'appropriation sans droit sont donc présents ici. Tout d'abord, l'absence de régulation entre les instances concernées, avec une nette supériorité des militaires sur les 
institutions telles que le gouvernorat et, a fortiori, la direction des Antiquités... Emporte l'usage du foncier celui qui détient finalement les rênes du pouvoir dans le pays, quelle que soit la situation juridique. L'autre élément est plus récent ; il s'agit de la confusion résultant de la privatisation des sols, suite au démantèlement de certaines propriétés de l'État.

9 L'absence de documents graphiques actualisés sur le site, qui est invariablement représenté vierge, est un des principaux éléments favorisant la confusion et permettant à chacune des parties de manipuler le débat. Lors d'un procès opposant les habitants et la compagnie de Ma'âdi, on s'aperçoit par exemple que les habitants de 'Izbat Khayr Allah, zone d'habitation d'émanation populaire bâtie sur le pourtour sud-est du plateau dans les années soixante, estiment se situer hors des limites du terrain acquis par la compagnie de Ma'âdi'. L'antériorité de leur résidence exclurait donc peut-être la légitimité de la requête.

Quant au gouvernorat, ses relations avec la société de Ma'âdi sont des plus ambiguës : en dépit de certaines résistances, très perceptibles lors du procès en cours, le gouvernorat pourrait aisément décider de régulariser la zone. Cette impression, déduite des procès-verbaux, est corroborée par un entretien réalisé en 1991 avec un des députés du secteur. L'obstination de la compagnie de Ma'âdi, qui persiste dans ses demandes d'éviction auprès de la force publique, est confortée dans les faits par l'absence de gestion institutionnelle des contradictions. Tandis que le quartier se développe de jour en jour - atteignant quelque 100.000 habitants -, le gouvernorat réglemente par omission. Ainsi ne réagît-il pas au processus de lotissement clandestin massif qui se met en place à partir de 1985, mais qui avait déjà débuté en 1978.

11 A IstabI 'Antar, les tentatives pour tirer une valeur d'échange de parcelles découpées sur un terrain théoriquement inconstructible mobilisent les énergies à tous les niveaux, à la base comme dans des sphères plus protégées. De là découlent des systèmes d'alliance pour l'accaparement des sols et de protections en chaîne qui contribuent à obscurcir une situation foncière déjà très confuse.

$12 \mathrm{Si}$, dans les modes d'urbanisation non réglementaires sur terrains privatifs, l'on rencontre nombre d'acteurs engagés dans ce processus, y compris au sein de l'administration (nos interlocuteurs de Dâr al-Salâm évoquent fréquemment la délivrance, après-coup, de permis de construire en échange de dessous-de-table conséquents), la situation est relativement sûre pour les acquéreurs et bâtisseurs, qui ne risquent guère l'éviction. Il n'en va pas de même dans l'occupation de terrains de l'État, situation conflictuelle s'il en est. Pour l'ensemble des acteurs mobilisés, en particulier pour les acquéreurs de lots ou squatters, il est nécessaire de déployer des tactiques, voire parfois de véritables stratégies foncières ${ }^{10}$. Cela est d'autant plus vrai que leur droit d'usage ${ }^{11}$ sur ces terrains n'est pas reconnu et que plane toujours la menace de l'éviction dans les quartiers non régularisés.

Une vision segmentée et plurielle de la propriété

13 La concurrence entre les règles et les institutions, ici, n'est ni contrôlée ni sanctionnée. Ce «pluralisme légal » consiste en une multiplicité de règles - écrites ou non - en compétition, règles qui ne semblent pas avoir été édictées dans un souci réel d'application et ne correspondent pas non plus à un projet national (faciliter l'accès au sol aux citadins démunis, prendre acte des carences de l'État en matière de construction de logements à faibles coûts, etc.). L'absence de projet commun au sein 
des instances dirigeantes, l'acceptation du laisser-faire en dépit d'un discours légaliste, conduisent en fait à une absence de régulation explicite qui rend caduques tant les règles existantes que les définitions de la propriétét ${ }^{12}$.

Cette absence d'ordre - au sens de régulation explicite - fait prévaloir une conception à la fois utilitaire et brouillée du droit d'usage de la terre. Un des paradoxes suscités par cette situation réside dans la production, chez les squatters ou lotisseurs occasionnels, d'un discours légitimant le fait que les terrains de l'État ne peuvent être vendus. Certains expliquent parfois même, lors des enquêtes, qu'il est interdit d'y " construire ». On verra plus loin comment les pratiques s'ajustent à cette perception. Examinons dès maintenant comment ces différents acteurs, institutionnels ou non, se situent les uns vis-à-vis des autres.

La compagnie de Ma'âdi dénonce avec vigueur les « empiétements » - tabadiyyât - sur ses terrains. On notera qu'à l'opposé, les habitants n'utilisent jamais ce terme mais celui de wad' al-yad, qui se réfère encore à l'idée d'une pratique traditionnelle. La société immobilière décrit les occupants comme des usurpateurs ou des "voleurs" conduits par des visées spéculatives. Aucune attention n'est prêtée à la situation d'exclusion de l'accès au sol des catégories les plus pauvres. D'ailleurs, pour se dédouaner, les dirigeants de la société affirment que les pauvres ne constitueraient qu'une frange marginale de cette population, légitimant ainsi leur volonté d'éviction pour concrétiser leur ambitieux programme. La présence sur le plateau, depuis bientôt dix ans, de plusieurs dizaines de milliers d'habitants ne change rien à leurs demandes d'éviction réitérées.

De leur côté, les habitants formulent diversement leur conception de la légitimité. Aux yeux des leaders du quartier, fortement mobilisés autour d'une lutte visant à la régularisation, l'État, au sens large, est considéré comme extérieur au problème. Ses bonnes intentions sont d'autant moins mises en doute que les leaders sont affiliés au PND (Parti national démocrate au pouvoir).

L'absence de critique ouverte du gouvernement par ces habitants - qu'ils soient squatters depuis le début, acquéreurs de seconde main, petits lotisseurs ou locataires est liée à une demande récurrente : la prise en compte de leurs besoins élémentaires, et ce, au nom d'une citoyenneté revendiquée. Ces revendications vis-à-vis de l'État trouvent rarement à s'exprimer en termes de droit $^{13}$. Un habitant : "Tout ce que nous demandons, c'est qu'on nous aide à acheter la terre pour pouvoir vivre comme tout le monde. » Un second: «Nous voulons être tranquilles. » Un troisième : «Nous avons beaucoup souffert. »

Enfin, une bonne partie des habitants rencontrés estime avoir suivi une incitation gouvernementale à l'installation sur les terres désertiques pour contrecarrer la transformation très préoccupante des terres agricoles en terrains à bâtir. Prenant délibérément au premier degré un discours officiel, les premiers installés s'en sont servi comme d'un catalyseur leur ayant permis de franchir le pas. Les propos d'une femme appartenant à une famille de squatters-bâtisseurs est symptomatique de ce qui m'a été rapporté sur ce registre. Le mode de justification des premiers occupants se situe entre la légitimation pure et simple de leur action et une réponse issue d'une vision populaire, qui fait contrepoids et donne une autre mesure au discours dominant :

«Tu savais qu'il était interdit de construire?

- Interdit? Non, je ne savais pas... Pourquoi ils ne l'ont pas dit au départ? Ils ont

seulement dit: "Éloignez-vous des terrains à cultiver, allez dans le désert et 
construisez!»

- Qui a dit cela?

- On a entendu ça à la télévision. Beaucoup de gens ont entendu ça. »

19 Ainsi, en répandant l'idée que le développement urbain doit se faire dans le désert, le gouvernement inciterait à l'occupation de parcelles assainies ou de logements déjà construits. Partant du constat que le rapport de forces entre les autorités et la population n'est pas en faveur de cette dernière, celle-ci opère un déplacement de la mobilisation. Ainsi, le cheikh qui mène l'essentiel de la lutte pour la régularisation reconnaît que « au lieu de nous bagarrer avec le gouvernement, nous avons eu recours à la justice. Nous ne sommes pas de taille à lutter contre le gouvernement ».

Mais les habitants ont eux aussi trouvé des cibles pouvant cristalliser leur mécontentement. En première ligne, la société immobilière de Ma'âdi, que les personnes les plus mobilisées considèrent comme l'unique responsable des maux qu'endurent les habitants. La lutte pour la régularisation étant menée par des leaders affiliés au PND, la critique est détournée. Très impopulaire, la société de Ma'âdi est désignée comme un agent de la spéculation, ses dirigeants sont taxés d'amoralité. C'est une société "vénale» et même «anti-égyptienne» (l'on vise ici la construction d'hôtels, et on prête également à cette société des réalisations telles que des casinos, ce qui reste à prouver).

21 L'interprétation de la légitimité du «droit foncier » révèle en outre une convergence étonnante entre les discours des adversaires les plus farouches : la société de Ma'âdi et les habitants. En effet, si les habitants non apparentés aux lotisseurs soumettent ces derniers à de vives critiques, seule la catégorie des lotisseurs " professionnels ", qui ont accaparé et morcelé le terrain en 1985, sont désignés comme étant nuisibles aux intérêts des habitants. Cependant, les appuis dont bénéficient les lotisseurs leur assurent une relative impunité. Exerçant en outre un contrôle sur la majeure partie des terrains et impliqués dans une démonstration de force permanente, de type mafieux, ils ont réussi à morceler et mettre en vente plus de la moitié des 200 hectares conquis sur le plateau. Pour ce faire, ils n'ont pas eu besoin de recourir au moindre subterfuge ni de passer de réel compromis avec les acquéreurs.

En revanche, les lotisseurs occasionnels, bien qu'agissant en groupe (il s'agit de la deuxième génération des habitants issus des contreforts du plateau) sont dépourvus d'appuis. Très bien acceptés par les acquéreurs de lots ou les squatters-bâtisseurs, ils sont obligés, pour se protéger, de tenir compte du caractère incessible des terrains de l'État mais aussi d'offrir une petite garantie aux acquéreurs. Après avoir saisi quelques terrains, ils édifient une baraque dans un coin. Tout se passe ensuite comme si seul cet édifice était revendu et non la terre, ce qui - en théorie -- peut paraître moins répréhensible.

Occuper n'est pas posséder

23 A travers quels dispositifs est contournée, mais aussi recomposée, la règle de nonaliénation des terrains de l'État? Les lotisseurs occasionnels, de même que les acquéreurs ou squatters amenés à mettre en vente leur parcelle (qu'ils visent la mobilité ou le bénéfice) revendent en fait un bâtiment. N'ayant pas, dans un sens, mis en vente le terrain nu, ils peuvent transmettre le bien avec une sorte de contrat, lequel instaure une relation de confiance entre des acteurs appelés à se côtoyer pendant la durée de leur résidence sur le site - alors que les lotisseurs professionnels, eux, résident bien souvent ailleurs. De part et d'autre, on s'accorde à dire que, sans 
reconnaissance de l'État, il s'agit d'un contrat symbolique. Cela rappelle, sans revêtir le même aspect coutumier, la pratique du contrat dit hujja en Jordanie, dans les quartiers urbanisés en dehors des règles légales ${ }^{14}$. Le contrat stipule que l'acquéreur achète " des briques» ou, le plus souvent, «des ruines (anqâd) sur les terrains de l'État». Pour expliciter les modalités de mise en œuvre de ces régulations, il faut revenir sur la façon dont les protagonistes décrivent et justifient la vente d'une propriété bâtie, et non du sol. On notera tout d'abord que la construction est souvent conçue comme provisoire. Lorsque la finalité de revente existe dès la mainmise initiale, la construction intervient pour permettre la réalisation du contrat. Les vendeurs occasionnels décrivent donc la vente de «ruines» comme une "vente construite?>. Le terme de "terrain» est clairement occulté. Ce qui est vendu en réalité, outre une petite pièce en parpaings avec un toit de canne à sucre et de paille, c'est un terrain beaucoup plus vaste que la surface occupée par cette simple pièce.

Un lotisseur occasionnel, aujourd'hui simple locataire d'un petit deux-pièces dans le quartier, relate cette pratique en ces termes : «Si on dit qu'on vend un terrain vide, on peut avoir un procès. Mais si on écrit sur le contrat qu'on a vendu "des ruines ", ça veut dire qu'on a vendu des bâtiments. Ça n'a rien à voir avec le terrain, même si les bâtiments se trouvent sur les terres de l'État. » Le ferme de " ruines », s'il fait référence au caractère précaire des premières constructions, est essentiellement destiné à masquer, en cas d'intervention répressive de l'État, le fait que l'on ait construit de manière définitive sur des terrains squattés. L'usage de ce terme révèle une tactique foncière - mais aussi une grande incertitude sur ce qui est autorisé ou non - qui, peu à peu, s'impose comme une pratique dominante des habitants, une règle d'usage à part entière. Il s'agit, à tout le moins, d'une terminologie de précaution.

La garantie d'un droit d'usage, mais aussi de transmission de ce droit aux ascendants, est donc ici en jeu. Il importe peu, dans cet esprit, que les titulaires de ce «droit» paient, leur vie durant, une indemnité d'occupation, à l'instar des titulaires de hikr. En revanche l'imposition, par les pouvoirs publics, de règles retirant aux occupants sans titre la jouissance de leur terrain, occasionne des pratiques nouvelles, porteuses d'un « contre-droit » dont les pouvoirs publics ont totalement perdu la maîtrise ${ }^{15}$.

En sortant du domaine des pratiques pour aborder celui des représentations, on s'aperçoit que les habitants de IstabI 'Antar ont sans conteste à l'esprit une perception idéalisée de l'habitat, de la ville, qui s'apparente de près ou de loin à l'urbanisation dense et verticale, aux immeubles bâtis sur des terrains privatifs. Lorsque les familles en quête de mobilité sociale désignent l'endroit ou elles souhaiteraient résider, il s'agit presque toujours de terrains ou de bâtiments soumis à la propriété privée. Or, dans ces quartiers, les prix des terrains, des pas-de-porte ou des appartements leur sont inaccessibles. Il existe un fossé important entre la grande majorité des habitants d'un quartier squatté et ceux de quartiers situés sur terrains privatifs.

La demande sociale des habitants est en réalité presque totalement déconnectée de cette représentation idéale. A un stade de capitalisation inexistant ou embryonnaire, les habitants cherchent une sécurité de tenure. Craignant de voir des années d'investissement ruinées par l'arrivée de bulldozers, comme ce fut le cas pour quelques habitations au tout début de l'implantation, ils veulent se protéger : tous expriment le souhait de "vivre en paix », de pouvoir enfin «se reposer ", de ne plus vivre dans la peur de l'éviction ou les difficultés d'obtenir une régularisation de fait, via l'arrivée des infrastructures de base. La privation d'accès à l'eau du réseau public est en effet ce qui 
grève le plus le budget, mais empêche aussi tout développement d'activités économiques.

Représentation populaire de la tenure foncière

II serait logique que l'occupation de terrains, mais aussi leur mise en valeur, serve de tremplin pour réclamer le droit à une tenure à plus ou moins long terme. Or, à aucun moment les habitants ne réclament la propriété de terrains de l'État, mais seulement la reconnaissance d'un droit d'usage. Il ne s'agit pas seulement d'une stratégie de contournement ou d'une tactique foncière. En premier lieu, la prédominance, depuis quelques années, de ce type de transaction en a imposé la pratique à tous, et tous semblent l'avoir intériorisée. L'occupation sans titre de terrains est d'autant plus rentrée dans les modes de répartition foncière des lotisseurs occasionnels, des acheteurs et des bénéficiaires à titre gratuit - généralement parents des lotisseurs que le respect pour les lois de l'État ou supposées telles est paradoxalement très fort. Les catégories du droit de propriété sont en fait réinterprétées à l'aune d'usages plus anciens. A côté du droit de propriété perpétuel (mulk), l'égypte connaît un type de tenure à bail sur des terrains qui sont propriété de l'État (hikr). A la différence du mulk, le hikr consacre le droit d'usage et non le droit de disposition ${ }^{16}$ moyennant un loyer annuel versé aux services de la propriété foncière des gouvernorats. De plus, le hikr n'est pas aliénable.

Il en était de même de la tradition de dissociation de l'usage du sol et de la propriété dans les villages égyptiens. Sans doute faut-il garder à l'esprit que la propriété privée des sols n'est apparue dans les campagnes égyptiennes qu'à la fin du xixe siècle. Jusquelà, la terre était la propriété du souverain, l'État répartissant la surface à cultiver en fonction de l'organisation du travail et de la planification décidée au sommet. Après la promulgation des deux dernières lois grevant les servitudes qui restaient sur la terre, une partie non négligeable de la paysannerie est devenue locataire de la terre ${ }^{17}$.

De nos jours, sur les terrains privatifs dont la propriété a été légalement transmise, l'ambiguïté peut également prévaloir. L'obtention d'un permis de construire juridiquement valide ne devient un enjeu que si l'on veut bénéficier de matériaux subventionnés. Le propriétaire se livre alors à un arbitrage subtil entre la somme qu'il devra débourser éventuellement en amendes, le temps que lui prendront les démarches et le dessous-de-table qu'il lui faudra bien souvent verser pour obtenir une dérogation.

31 La vision étatique des divers types de statut foncier repose schématiquement sur une opposition entre droits de propriété et occupation sans droits. L'apparente irréductibilité de ces deux catégories accentue, dans les pays où la généralisation du droit de propriété est récente, des formes de discrimination sociale. Le «droit à la ville» est en réalité accordé aux seuls détenteurs d'un droit de propriété foncière en bonne et due forme, alors même que l'accès à ces terrains est fortement réduit par l'appareil d'État : soit parce que les terres sont inégalement enregistrées, soit parce que les terrains privatifs, comme en Égypte, sont en grande partie inconstructibles (terres agricoles).

32 Enfin, sous certaines conditions, la loi reconnaît le principe de la prescription pour des terrains mis en valeur ${ }^{18}$ par ses occupants, même si ces derniers l'étaient sans titre. A des dispositions contenues de longue date dans le code civil se sont greffés d'autres instruments législatifs permettant d'assurer la régularisation foncière sous certaines conditions. 


\section{La régularisation}

C'est à travers celle-ci que s'affrontent les diverses représentations de la propriété, de la possession du sol et aussi de l'espace que l'on s'adjuge. Il existe d'abord un substrat juridique, qu'il nous faut examiner pour connaître ses interprétations à la fois dans les sphères du pouvoir et dans celles de la société civile. En matière d'occupation sans titre de terrains, la loi égyptienne, à l'instar de celle de nombreux pays arabes et en vertu d'une pratique qui remonte à la Rome antique, concède la prescription sur des terrains occupés illégalement, mais mis en valeur. Autrement dit, les occupants sans titre deviennent d'emblée propriétaires s'ils peuvent prouver qu'ils ont occupé et valorisé un terrain de façon continue et sans contestation du propriétaire, cela durant quinze ans.

Ce régime juridique, celui du wad' al-yad (mainmise), définit aussi une pratique puisque les occupants sans titre et squatters disent avoir "posé la main» sur un terrain. Ils savent donc pouvoir bénéficier à terme de l'application de cette législation. Cependant, une loi édictée, à l'origine, dans un souci de régulation de pratiques agro-pastorales basées sur l'usage extensif du sol ne peut facilement être transférée dans le champ urbain, surtout lorsque la pratique du wad' al-yad se répand. Si des régularisations se sont produites au coup par coup sous l'impact de cette norme juridique, cela a été très loin d'être systématique. La possibilité de bénéficier du régime de la mainmise est par ailleurs fortement tempérée par l'article 970 du Code civil, qui exclut du bénéfice de la prescription les occupations sans titre de biens appartenant à l'État et les biens détenus par des organismes publics ${ }^{19}$.

La politique d'ouverture économique lancée par le président Sadate dans les années soixante-dix a ainsi ouvert la brèche à un démantèlement partiel des propriétés de l'État. Ainsi, depuis 1984, une loi autorise les gouvernorats à transférer un terrain propriété de l'État - à des occupants sans titre, s'ils occupaient le terrain antérieurement à cette date. Si cette loi traduit une certaine ouverture législative, due largement à une pression externe (celle de l'USAID) pour ouvrir le marché foncier, elle a aussi pour finalité de drainer des ressources publiques dans les gouvernorats. Mais l'apparition de cette règle de droit est venue se superposer à celle qui régit la prescription, créant la confusion chez les personnes susceptibles de bénéficier de l'un ou l'autre des régimes.

Ainsi, alors que ces possibilités de régularisation ne s'appliquent pas, en principe, aux terrains occupés après 1984, les habitants en revendiquent le bénéfice. En pratique, ils en sont exclus, bien qu'étant les principaux intéressés. Dix ans après la promulgation de cette loi, il n'y a toujours pas d'application. Dans l'ensemble, les régularisations effectuées n'obéissent ni aux règles édictées ni aux besoins réels de la population. Dans les cas d'occupations de terres du domaine de l'État, ce sont les rapports de force et l'intervention d'institutions transnationales qui déterminent finalement la régularisation. Ainsi en a-t-il été de la régularisation du quartier de Manshiyat Nasser, englobé dans un vaste programme de restructuration urbaine sous l'impulsion de la Banque mondiale. Dans le cas de 'Izbat al-Haggana, ce sont les militaires qui peuvent accorder une régularisation prenant la forme d'un rachat de titres fonciers. Enfin, à Istabl 'Antar, le procès de longue haleine mené par un groupe d'habitants infatigables et l'appui d'avocats et de députés du secteur ont sans doute contribué à ce qu'une certaine sécurité foncière commence à voir le jour dans les années quatre-vingt-dix. Elle se manifeste, de facto, par un accord enfin explicite de la compagnie distributrice 
d'eau pour raccorder l'ensemble des habitations au réseau (moyennant des prix de raccordement très élevés) au lieu de procéder au coup par coup.

Alors que le devenir du quartier semble toujours menacé, la régularisation a avancé à grands pas grâce à la mise en route récente d'un programme d'amélioration de l'habitat. La construction et la consolidation se sont nettement intensifiées, des responsables de secteur ont été nommés, une école est en voie d'édification.

Genèse des représentations de l'espace nouvellement urbanisé

Dans cette seconde partie, je confronterai les "espaces de représentation " et les représentations de l'espace chez les classes populaires et chez les planificateurs, et je tenterai de montrer les effets de cette rencontre sur la production de l'espace.

Le plateau de Fustat ${ }^{21}$ se présente comme une marge encerclée de tissus urbains disparates et très densément peuplés. Tout le secteur environnant de Masr al-Qadîma ou d'al-Khalîla est urbanisé ou occupé (notamment par des nécropoles) depuis des millénaires. A partir des années soixante, l'urbanisation s'est étendue au bassin agricole que l'on appelle aujourd'hui Dâral-Salâm, vaste quartier d'émanation populaire sur terrains privatifs, et a peu à peu encerclé le plateau. On a vu surgir, sur des terrains du domaine de l'État, des constructions en torchis disposées sur une trame très serrée.

C'est à la lisière des quartiers de Dâr al-Salâm et Basatîn ${ }^{22}$ que s'élèvent les flancs du plateau de Fustat. Emprise très étroite, certes, mais ni cultivée ni privative, et inclue dans le périmètre indéterminé des possessions du gouvernorat au même titre que le plateau. L'implantation dans ces secteurs grignote de l'espace en attaquant les falaises qui entourent le plateau. Les habitations sont construites le long de couloirs récemment gagnés sur le massif rocheux, suivant une topographie très heurtée.

41 Au milieu des années quatre-vingt, une ou deux nouvelles générations sont nées dans ces quartiers aux toponymes variables 'lzbat Khayr Allah, al-'lzba al-Qadîma, IstabI alHaggana, Wadi 'Antar. Manshiyat Amr, 'Izbat al-Tahawi). Ces quartiers sont saturés, l'extension horizontale et l'extension verticale étant arrivées à leur maximum. Personne ne dispose de ressources permettant de construire en armature de béton, et la topographie limite considérablement la verticalisation.

Ce sont les ménages habitant déjà le secteur de Fustat qui, par nécessité de reproduction externe ${ }^{23}$, vont être les premiers à envisager de s'installer sur cette face du plateau. Dépourvu de constructions au début des années quatre-vingt, le secteur sud du plateau fait alors office de front pionnier, un des derniers du Caire avec les hauteurs du Muqattam. L'usage de l'expression « front pionnier » est ici d'autant moins usurpé que tout, dans le récit des "colons", traduit des efforts considérables de mise en valeur.

Exemple paradoxal de site quasiment dépourvu d'habitations en plein Caire, ce plateau est resté longtemps protégé des empreintes urbaines tant il était difficile d'accès (anciens tracés, dénivelés impressionnants, mais aussi protection foncière due à son statut de site archéologique). A en croire les récits des habitants, il aurait dû rester vide. II est en effet dépeint, avant son occupation, comme un "désert» ou une « montagne » où règnent d'effrayantes créatures (djinns, loups, esprits, fantômes...) Les nouveaux arrivants d'istabl 'Antar évoquent leur rejet du plateau avant son urbanisation. Qu'il ait été zone de fouilles archéologiques n'enlève rien à cette perception dominante. Quant aux personnes s'étant récemment installées à la 
périphérie, elles témoignent d'une double influence culturelle : une socialisation dans un univers densément peuplé, mais aussi une conception du monde encore partiellement ancrée dans l'espace-temps propre à la paysannerie égyptienne ${ }^{24}$.

Même si elle s'applique ici à l'espace interstitiel, la partition binaire existant fréquemment dans les représentations populaires des sociétés "traditionnelles » en matière d'espace ne semble guère altérée. Dans cet esprit, l'espace naturel est assimilé au désordre, à l'état sauvage, et l'espace humanisé identifié à l'habitat ${ }^{25}$. Cela recoupe, on le voit, la distinction entre urbanité et état de nature. Enfin, en Égypte, l'espace cultivé rentre, semble-t-il, dans la catégorie de l'espace humanisé, compte tenu de la profonde emprise humaine sur la terre comme sur la répartition de l'eau, et ce, depuis des millénaires. En revanche, est qualifié de désert, donc assimilé au non-humanisé, tout espace non mis en valeur. Sans risquer d'interprétation figée, on essaiera de comprendre ces classifications de l'espace à travers une démarche comparative. La forêt - autre espace non humanisé - est perçue de la façon qui suit par les immigrants dans le port naissant de San Pedro, en Côte-d'ivoire : « En partie inondée et regorgeant donc d'humidité et de moustiques, cette foret est évidemment un milieu répulsif... Mais surtout, elle est la négation du fait urbain, et elle est tout particulièrement ressentie comme telle dans cette contrée du bout des terres, jusqu'ici impénétrée, que l'immigrant a hâte de voir s'humaniser et se structurer : besoin et désir de voir se développer une vie de relations de caractère urbain, propre à faire oublier l'environnement peu hospitalier et à rassurer quant à la réalité d'une ville sur laquelle on a reporté tous ses espoirs. " ${ }^{26}$ De même, dans les villages kabyles des années cinquante, subsistait la croyance selon laquelle on s'exposait à l'extinction de la famille si l'on construisait en dehors du village ${ }^{27}$.

Compte tenu de l'urbanisation ancienne et de la centralisation très forte qui a toujours régné en Égypte, les exemples cités plus haut ne doivent pas être pris dans leur sens littéral. Ils viennent illustrer la convergence des codes que se donne une société en partie dominée, celle des exclus du marché du logement officiel, celle d'habitants qui se voient privés de sécurité foncière et n'ont qu'un accès très limité aux sphères du pouvoir. La représentation du monde politique et de l'espace chez une population presque totalement analphabète ne correspond donc pas aux canons de la géométrie euclidienne et de l'espace abstrait, anonyme, en trois dimensions. Cependant, ce n'est pas d'une non-représentation qu'il s'agit mais d'une autre lecture de l'espace, qui s'opère par le biais de codes récurrents, communs aux membres de cette collectivité ; une lecture qui donne une raison d'être et fait partager les mêmes valeurs.

On le comprendra, ces représentations s'opposent à ce que le site soit mis en valeur. Le déblocage de la situation foncière ne peut se comprendre sans la raison objective que constitue la mise en vente du site, traduisant l'ouverture ou la déréglementation des marchés fonciers publics. Cette mise en vente a suscité, on l'a vu, une concurrence foncière permettant aux lotisseurs et squatters de tirer profit de la vacance juridique. Mais cette libération de la terre et son occupation ont eu besoin, semble-t-il, de s'appuyer sur un deuxième vecteur d'urbanisation : les lotisseurs. Ce sont eux en effet - qu'ils soient professionnels ou occasionnels - qui ont ouvert le terrain à l'urbanisation en occupant l'espace, en le remplissant. Ils ont ainsi assuré une double fonction de protecteurs: symboliquement, contre les «forces magiques» qui peuplaient le plateau ; concrètement, vis-à-vis des autorités. 

mythe de fondation. Aussi le peuplement de ces quartiers est-il largement surestimé. Là encore, comme dans la pratique des anqâd, il ne s'agit pas seulement d'une stratégie destinée à faire plier le gouvernement. Si cette intention existe, la surestimation systématique a probablement aussi pour vocation de contrebalancer la vision d'un espace vide, donc vécu comme dangereux. L'urbanisation du plateau est perçue comme un processus d'agglomération, et son achèvement est lié à la densification : "Les gens habiteraient ensemble, jusqu'à ce que la montagne se remplisse.» "La montagne deviendra belle lorsque elle sera construite, couverte d'immeubles.» Par bien des aspects donc, l'espace n'est pas dissociable d'un projet social lié au « faire ensemble »et non pas «fait pour»-, donc centré autour de l'idée de collectivité. Dans un deuxième temps apparaît la préoccupation liée au remplissage de ce vide : il s'agit de le meubler, de l'aménager, ce qui revient à le construire.

n aura ainsi tout un jeu d'équivalences et d'oppositions utilisées dans les descriptions du site ou de la situation Juridique. Ces systèmes d'opposition viennent ainsi conforter l'habitant-bâtisseur dans l'idée que le quartier est urbanisé, dense, donc inamovible. Il ne s'agit plus là d'un mythe de fondation mais de consolidation, dont une des fonctions serait de révéler aux yeux du monde l'importance du peuplement dans ces zones peu ou pas reconnues ${ }^{28}$. Un habitant-bâtisseur de IstabI 'Antar : « Bien sûr, le gouvernement ne peut pas expulser deux ou trois millions de personnes installées sur 250 feddans, ou à peu près. Pas seulement les 25 feddans proches d'ici, mais plus que cela (décrivant) : il y a le désert, immense, étendu. Ensuite, il y a des bâtiments récemment construits, et parmi eux, certains sont de la meilleure qualité. »

L'appréhension des grands nombres

La population du quartier, pour des raisons liées à la division administrative des secteurs du recensement, n'est pas connue .officiellement. Dans ma thèse, à partir de recensements partiels effectués par la population et d'extrapolations, j'obtiens le chiffre (en 1991) de 47.000 habitants pour IstabI 'Antar (plateau et contreforts) et de 53.000 pour Izbat Khayr Allah.

Les habitants se sont en effet abonnés en grand nombre à l'électricité et ont réalisé un recensement partiel pour prouver, lors des procès divers, la matérialité de leur existence. Un des leaders du quartier, à partir du chiffre de 8.650 bâtiments connectés (soit environ la moitié du total), évalue la population totale du secteur entre 500.000 et un million d'habitants ${ }^{29}$.

51 La représentation du peuplement qui prévaut est commune aux habitants et à certains agents de la fonction publique travaillant sur les questions urbaines. Ainsi, la population de Manshiyat Nasser, qui atteignait en 1986130.000 habitants, est évaluée à 1 million d'habitants par un responsable du service des domaines du gouvernorat; Ces représentations s'enracinent dans l'expérience quotidienne de la densité, l'espace tirant ainsi sa substance des éléments de l'existence ${ }^{30}$ mais aussi de la vision largement fantasmatique d'un exode rural incontrôlé, submergeant la ville. Ainsi nous a-t-on dit que la population de Manshiyat Nasser pouvait effectivement avoir augmenté de quelque 800.000 personnes en cinq ans, étant donné l'ampleur de l'arrivée de nouveaux immigrants. C'est seulement la frange la plus jeune et instruite qui pensera en termes d'équipements futurs et dépassera l'idée de «saturation de l'espace ». Conclusion

Égypte/Monde arabe, 23 | 1995 
Réfléchir sur les normes véhiculées par les acteurs institutionnels et les habitants, cela suppose de rappeler et d'explorer en profondeur les catégories de perception et d'action de ces derniers. En termes opérationnels, cela peut alimenter le débat sur la régularisation foncière, dont on a vu avec quelle lenteur elle progressait, paralysée par l'absence de volonté politique affirmée face à des squatters mal acceptés et à des enjeux économico-politiques sur le foncier.

Les questions abordées ici permettent également d'affiner l'approche souvent globale et externe que nous avons de la ville en mouvement, et de lire les pratiques sociales à la lumière de leur caractère souvent conflictuel.

54 La multiplicité des enjeux et statuts fonciers, l'opacité des règles et la recomposition souvent peu extériorisée des acteurs va à mon sens à rencontre de l'analyse déterritorialisée, de la généralisation à partir de caractéristiques physiques apparemment similaires et dont on extrapole une série de convergences d'ordre social, économique, voire juridique.

Ainsi en est-il des développements sur «l'uniformité du Caire ». De ces périphéries densément peuplées et construites découle l'idée de territorialisation à l'identique. Si la morphologie des bâtiments semble uniforme, elle l'est surtout parce qu'elle dénote un mode d'articulation original entre promotion foncière et immobilière, de type petitmarchand. Mais ce constat dispense trop souvent de concevoir la diversité urbaine audelà de l'apparente uniformité. Les jeux des acteurs sont loin d'être équivalents d'un quartier à l'autre. Le Caire, y compris dans la rencontre entre la «ville légale » et la "ville d'émanation populaire», est une agglomération plurielle, non duale. On peut ainsi repérer des éléments de différentiation interne aux espaces urbanisés : statuts fonciers imprimés par le gouvernorat, réinterprétations locales et perception des hiérarchies urbaines impriment leur marque à ces territoires.

Prenons le cas de Dâr al-Salâm, avec ses différentes unités. Derrière le qualificatif général d'« informel », derrière l'unité visuelle due à l'abondance des bâtiments en structure poteau-poutre, remplissage de briques, non crépis, on retrouve des «territoires » sous le «territoire " ${ }^{31}$. Comme Dâr al-Salâm, IstabI 'Antar n'est réductible ni à un type de peuplement homogène ni à un mode d'occupation de l'espace ou à une conception urbaine unitaire. C'est une des configurations possibles d'un système local de l'habitat, travaillé en profondeur par les formes dominantes de production. Son devenir, y compris foncier, est fortement conditionné par le projet de voie périphérique qui devrait prochainement entraîner la démolition de plusieurs centaines d'habitations. Cette solution radicale - alors qu'un contournement serait possible n'a guère été contestée par les médias, à l'opposé de ce qui s'est passé lors de la construction du tronçon de Basâtin. Les projets d'aménagement actuels (éradication de certains quartiers, rénovation urbaine, voie périphérique) ne sont-ils pas contradictoires avec le credo de la régularisation? 


\section{NOTES}

1. Cet article est en partie tiré de : Vers un urbanisme d'émanation populaire. Compétences et réalisations des citadins. Le cas du Caire, Thèse de Doctorat, IUP, Université de Paris XII, 1994.

2. L'expression est de J. F Tribillon (Villes africaines. Nouveau manuel d'aménagement foncier, AOEF, 1993). L'auteur définit (p. 24) le « droit populaire » ou la « nouvelle coutume urbaine » par opposition au « droit coutumier » ou au « droit de l'administration »: « En ville se développe un nouveau droit que l'on peut dire synchrétique mais dont la composante coutumière est la plus forte, à tel point que l'on est fondé à parler d'une nouvelle coutume urbaine. C'est une sorte de droit immanent que pratiquent "naturellement" les gens ordinaires. »

3. Pinson D., Modèle d'habitat et contre-types domestiques au Maroc, Urbama, fascicule de recherche $\mathrm{n}^{\circ} 23$, Tours, 1992, $223 \mathrm{p}$.

4. Cette notion est tirée de la thèse de $0 . \mathrm{M}$. Razzaz, Law, Urban Land Tenure and Property Disputes in Contested Settlements: the Case of Jordan, PhD soutenu à l'université de Harvard, mai 1991.

5. Cette appellation recouvre aujourd'hui les terres non cultivées (désertiques en particulier) placées, selon le cas, sous la responsabilité du ministère de la Construction et du Logement ou sous celle des gouvernorats. Dans la catégorie des terrains non privatifs entrent également ceux dits « du domaine public », terrains utilisés à des fins d'équipement, de transport ou à des fins militaires. Ces derniers ne peuvent être rétrocédés à des particuliers.

6. Deboulet A., «Squatters, État et maîtrise de l'espace au Caire ", Égypte/Monde Arabe $\mathrm{n}^{\circ} 1,1990$, p. 79-95.

7. A cet égard, la situation n'est pas très éloignée de celle qui prévaut à ' Izbat alHaggana. Voir à cet égard la thèse de magistère soutenue par Farag Abd al-'Aziz Sa'id, Projet d'amélioration de 'Izbat al-Haggana (en arabe), Département d'urbanisme de l'université du Caire, 1988-89.

8. Istabl 'Antar englobe aussi le sous-quartier de 'lzbat Khayr Allah : aujourd'hui, les espaces libres entre les deux zones se sont progressivement comblées sous la progression de l'urbanisation.

9. Sur le plan représentant le projet d'urbanisation de la société de Ma'âdi, le tracé des pourtours du projet montre bien, à la limite des terres agricoles et du plateau - au sudest de ce dernier -, une partie se trouvant hors de la zone cédée à la société. S'agissant de terrains arides, en partie gagnés sur les flancs rocheux, la partie de ce secteur dit de 'Izbat Khayr Allah peut être considérée comme relevant du domaine de l'État (du gouvernorat en l'occurrence).

10. lci s'impose la référence à l'ouvrage de P. Canel, Ph. Delis, Ch. Girard (Construire la ville africaine, Karthala-ACCT, 1990, 187 p.), qui décrit abondamment la « sémiotique foncière " manifestée par les habitants-constructeurs face à la situation de " pagaille » foncière qui règne à tous les niveaux à Kinshasa : les ruses des habitants, les stratégies des acteurs plus influents, lotisseurs...

11. L'occupant sans titre tire en effet profit, comme le montre S. Lechtimy dans le cas martiniquais (De l'habitat précaire à la ville. L'exemple martiniquais, L'Harmattan, coll. Objectif ville, 1992), d'un bien dont la nue-propriété appartient à autrui. 
12. Sur la notion de règles, je m'appuie notamment sur l'essai de J. D. Reynaud, Les règles du jeu. L'action collective et !a régulation sociale, Armand Colin. 1993.

13. En fait il s'agit, à l'instar de ce que montre 0 . M. Razzaz, de la différence entre legal rights et legal claims, dont le champ d'application et la signification peuvent être tout à fait distincts.

14. 0 . M. Razzaz retrace l'histoire de ce type de contrat, qui était déjà requis à l'époque ottomane lors des transactions foncières. Ce n'est que le Tandhimât qui instaura, en plus, le document de vente. Le contrat dit hujja (preuve) n'a juridiquement aucune valeur, mais à force d'être maintenu entre les parties contractantes (ne possédant pas légalement la terre), sa pratique s'est imposée comme un engagement du vendeur pour protéger l'acheteur. Cet engagement est mentionné explicitement dans le contrat. 15. Sur ce sujet, voir l'article de F. Drosso, « Le Caire : décalages entre la loi el les pratiques urbaines ", Les Annales de la Recherche Urbaine n³8, juin-juillet 1988, p. 80-85. L'auteur écrit (p. 81) : "Tourner la loi de l'État ne devient-il pas la quasi-loi de la ville? En anticipant sur l'imagination urbaine, en prévoyant ses prochains lieux de débordement, l'Étal ne désignerait-il pas, par son volontarisme, les territoires à occuper ? Une certaine complicité unirait alors ce couple antagoniste dans une coproduction de la ville interdite. "

16. Pour reprendre une distinction faite par E. Le Roy dans l'ouvrage collectif de E. Le Bris, E. Le Roy et P. Mathieu, L'appropriation de la terre en Afrique noire, Karthala, 1991.

17. Voir à ce sujet J. Lozach, Le Delta du Nil. Étude de géographie humaine, Le Caire, 1935.

18. On laissera de côté dans cet article le cas des waqf (pl. awqâf), ou biens de mainmorte, gérés par le ministère du même nom.

19. Article 970 : «Il ne convient pas de soumettre à la propriété les biens de l'État ou des personnes morales publiques, ni ceux des unités économiques dépendant des organismes publics et des compagnies du secteur public, ni les awqâf de bienfaisance, pas plus qu'il ne convient d'en tirer bénéfice par prescription. Il ne convient pas d'attenter à ces biens. En cas de transgression, il appartient au ministre concerné d'intervenir ainsi qu'il en a le droit. »

20. Suivant la terminologie employée par H. Raymond dans L'architecture, les aventures spatiales de la raison, CCI, Paris, 1984. L'auteur désigne par là l'ensemble des outils, symboles et idées permettant la représentation de l'espace dans un contexte socioculturel donné : le plan, le graphisme utilisé dans telle culture, etc.

21. Ce plateau a vu s'implanter la première ville musulmane d'Égypte en 647.

22. Tous deux inclus aujourd'hui dans le qism de Basatîn.

23. J'utilise ici l'expression de J.-C. Driant, qui explique comment les liens de type communautaire et les liens de parenté sont maintenus sur une base volontariste par les ménages des barriadas de Lima, en dépit de la saturation résidentielle. Lorsque la reproduction " interne ", c'est-à-dire l'installation des enfants devenus indépendants, ne peut se faire sur place, l'auteur parle de reproduction externe (J.-C. Driant, Consolidation et reproduction des quartiers populaires. Le cas des barriadas de Lima, Thèse de doctorat, Institut d'urbanisme de Paris, 1989).

24. Cette dernière proposition n'a pu être approfondie. Elle nécessiterait sans doute un échange entre sociologie urbaine et sociologie rurale.

25. Lévy F.-P., Segaud M., Anthropologie de l'espace, éd, du CCI, 1983, 329p.

26. Haeringer P., « San Pedro, la première vague d'immigrants », Cahiers de l'Orstom, série sciences humaines, vol. X, n²/3,1973, p. 259.

27. Bourdieu P., Esquisse d'une théorie de la pratique, Droz, Genève, 1972. 
28. Cette dernière partie du texte a fait l'objet de discussions avec Habib Ayeb, dont certaines critiques ont été fort éclairantes.

29. A partir du même chiffre, et en évaluant le nombre de maisons vides, nous avons effectué un calcul pondéré suivant le nombre de membres par ménage. Nous sommes arrivés à un total (plateau et pourtours) de 97.000 habitants.

30. Boughali M., La représentation de l'espace chez le Marocain illettré. Mythes et tradition orale, Anthropos, 1974.

31. Ce thème a été développé en particulier aux journées doctorales d'Urbama (Tours), en novembre 1994.

INDEX

Mots-clés : urbanisation, Le Caire, informel, habitat, ville, régularisation

\section{AUTEUR}

AGNÈS DEBOULET

École d'Architecture de Nantes/Urbama 